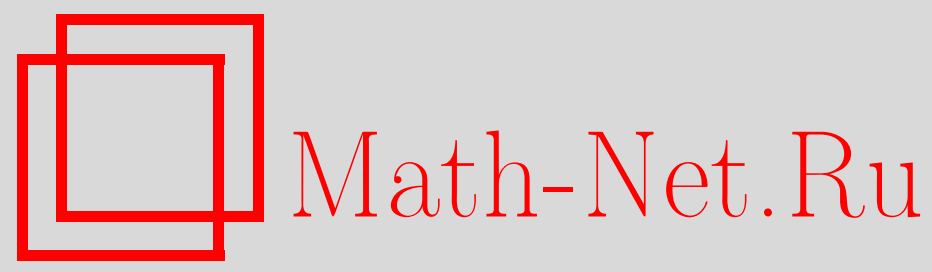

С. В. Рыков, О свойствах генератора, Дискрет. матем., 2011, том 23, выпуск 1, 51-71

DOI: https://doi.org/10.4213/dm1130

Использование Общероссийского математического портала Math-Net.Ru подразумевает, что вы прочитали и согласны с пользовательским соглашением http://www . mathnet.ru/rus/agreement

Параметры загрузки:

IP : 52.6 .47 .48

26 апреля 2023 г., $13: 22: 36$ 
УДК 519.7

\title{
О свойствах генератора
}

\author{
() 2011 г. С. В. Рыков
}

Генератор псевдослучайных чисел (ГПСЧ), порождаемый преобразованием

$$
F_{c}(x)=x+\left(x^{2} \vee c\right) \quad\left(\bmod 2^{n}\right),
$$

был представлен Климовым и Шамиром в 2002 г. Функция $F_{c}(x)$ транзитивна по модулю $2^{n}$ тогда и только тогда, когда $c \equiv 5(\bmod 8)$ или $c \equiv 7(\bmod 8)$.

В работе рассматриваются свойства распределения пар $\left(x_{i}, F_{c}\left(x_{i}\right)\right)$ ГПСЧ при всевозможных значениях $c \in \mathbf{Z} / 2^{n} \mathbf{Z}$ и показываются их неудовлетворительные статистические свойства, в особенности при $c \geqslant 2^{n / 3}$.

Показано, что в случае если $n=32$ требуется не более 9 различных пар $\left(x_{i}, F_{c}\left(x_{i}\right)\right)$ для определения значения $c$ с вероятностью $P \geqslant 0,999$.

\section{1. Введение}

Задачи построения и анализа генераторов псевдослучайных чисел (ГПСЧ), обладающих заданными статистическими, сложностными и иными характеристиками, встречаются в различных областях науки, где требуется источник псевдослучайных данных.

Особенные требования предъявляются к ГПСЧ, используемым в криптографии для построения быстрых и стойких поточных шифраторов, причем требование к быстроте является критическим, так как основная область применения поточных шифраторов это шифрование каналов связи. Для эффективной программной и аппаратной реализации шифратор должен быть изначально спроектирован в соответствии с выбранной архитектурой процессора/платы, набором поддерживаемых инструкций и рядом других условий.

Компоненты поточных шифраторов могут быть представлены композицией элементарных машинных команд. Наборы инструкций, поддерживаемые современными процессорами, постоянно расширяются, но в то же время практически каждый процессор поддерживает базовые логические (AND, OR, XOR, NOT, . . ) и арифметические операции $(+,-, \cdot, /, \gg, \ll, \ldots)$, проводит вычисления по некоторому модулю $2^{n}$ (например, $2^{8}$, $2^{32}$ или $2^{64}$ ). Заметим, что далее для обозначения машинных операций AND и OR будут использоваться $\wedge$ и $\vee$ соответственно. Иными словами, в некоторых случаях все эти операции можно рассматривать как преобразования в некотором базисе в кольце целых чисел $\mathbf{Z} / 2^{n} \mathbf{Z}$. Если из числа этих операций убрать несовместимые преобразования, такие как циклический сдвиг и сдвиг в сторону младших разрядов, то эти операции и любые их композиции будут также являться совместимыми [1].

Определение 1. Функция $f: \mathbf{Z} / p^{k} \mathbf{Z} \rightarrow \mathbf{Z} / p^{k} \mathbf{Z}$ называется совместимой, если для любых $a, b \in \mathbf{Z} / p^{k} \mathbf{Z}$ таких, что $a \equiv b\left(\bmod p^{k}\right)$ справедливо сравнение $f(a) \equiv f(b)\left(\bmod p^{k}\right)$, где $p$ - простое и $k$ - натуральное число. 
Данный класс функций представляет особый интерес в криптографии, так как любое совместимое преобразование представимо как композиция элементарных машинных операций, что позволяет создавать новые криптографические примитивы с определенными характеристиками, имеющие эффективную программную реализацию.

С начала 1990-х В. С. Анашиным [1, 3, 4, 5, 6] активно проводятся исследования совместимых преобразований с использованием методов неархимедова анализа. Рассматриваются свойства преобразований $f: \mathbf{Z}_{p} \rightarrow \mathbf{Z}_{p}$, удовлетворяющие условию Липшица с константой 1 , являющиеся продолжением совместимых преобразований с $\mathbf{Z} / p^{n} \mathbf{Z}$ на $\mathbf{Z}_{p}$ (см. раздел 3.8 .1 в [6]).

Определение 2. Функция $F: \mathbf{Z}_{p} \rightarrow \mathbf{Z}_{p}$ удовлетворяет условию Липшица с коэффициентом $k \in \mathbf{N}$, если для любых $a, b \in \mathbf{Z}_{p}$ выполняется неравенство

$$
|F(a)-F(b)|_{p} \leqslant k|a-b|_{p},
$$

где $p$ - простое число.

В серии работ Климова и Шамира начала 2000-х [12, 13, 14, 15, 11] совместимые преобразования рассматривались в частном случае при $p=2$. Введенное авторами определение $T$-функции по сути является сокращением от другого названия этого класса преобразований, известного из теории булевых функций, - преобразования треугольного вида.

Основные теоретические результаты, сформулированные в этих работах, были описаны еще в середине 1990-х [1, 2]. К практическим результатам, получившим широкое распространение, можно отнести примеры совместимых преобразований от одной и нескольких переменных. Некоторые из них были напрямую использованы при разработке алгоритмов поточного шифрования (как, например, семейство поточных шифраторов TSC) или предложены в качестве независимых компонент. К числу последних относится генератор псевдослучайных чисел TF-0, впервые описанный в работе [12]:

$$
x_{i+1}=F_{c}\left(x_{i}\right)=x_{i}+\left(x_{i}^{2} \vee c\right): \quad \mathbf{Z} / 2^{n} \mathbf{Z} \rightarrow \mathbf{Z} / 2^{n} \mathbf{Z} .
$$

На прошедшем в 2004-2008 г. европейском конкурсе поточного шифрования eSTREAM из 35 представленных 5 алгоритмов (ABC, Mir-1, MV3, TSC, VEST) были основаны на T-функциях. Отметим, что уязвимости в основном были обнаружены в функции инициализации, основная задача которой генерировать на основе ключа и вектора инициализации начальное заполнение регистров шифратора. На основное преобразование, вырабатывающее гамму и обновляющее свое внутреннее состояние, эффективных атак (за исключением младших модификаций TSC-1, TSC-2) не было найдено. Вследствие чего актуальны исследования, посвященные методам анализа шифраторов, основанным на Т-функциях.

В данной работе рассматриваются некоторые подходы к анализу распределения последовательных пар заполнений регистров $\left(x_{i}, x_{i+1}\right)$ основного преобразования поточного шифратора на примере ГПСЧ Климова-Шамира TF-0. В общем случае состояние $x_{i}$ может рассматриваться и как один из регистров, так и независимо как внутреннее состояние ГПСЧ.

В разделе 2 рассмотрены известные работы, посвященные криптоанализу $F_{c}(x)$. Раздел 3 показывает наличие линейных и квадратичных зависимостей в распределении пар $\left(x_{i}, F_{c}\left(x_{i}\right)\right)$ при произвольном фиксированном значении $n$ и различных значениях $c \in \mathbf{Z} / 2^{n} \mathbf{Z}$, раскрывающие неудовлетворительные статистические свойства генератора практически при всех значениях $c$. 
В [6] проводится параллель между автоматами Мили и совместимыми $p$-адическими преобразованиями, и показывается, что всякую совместимую функцию $g: \mathbf{Z}_{p} \rightarrow \mathbf{Z}_{p}$ можно рассматривать как автоматную функцию $\Psi_{\text {U }}$, соответствующую некоторому автомату Мили $U=\left\langle\mathscr{K}, \mathcal{N}, \mathcal{M}, f, F, u_{0}\right\rangle$, где $\mathscr{K}-$ входной алфавит, $\mathcal{N}-$ непустое множество состояний, $\mathcal{M}$ - выходной алфавит, $f: \mathscr{K} \times \mathcal{N} \rightarrow \mathcal{N}-$ функция переходов, $F: \mathscr{K} \times \mathcal{N} \rightarrow \mathcal{M}-$ функция выхода. Если задать входной и выходной алфавиты равными некоторому алфавиту $\mathscr{P}$, состоящему из $p$ символов, то можно установить взаимно однозначное соответствие между автоматной функцией $\Psi_{\imath}$ и $g$.

Определение 3. Будем говорить, что совместимой функции $g: \mathbf{Z}_{p} \rightarrow \mathbf{Z}_{p}$ соответствует автомат Мили $U=\left\langle\mathcal{K}, \mathcal{N}, \mathcal{M}, f, F, u_{0}\right\rangle$, если существует взаимно однозначное соответствие между автоматной функцией $\Psi_{\varkappa}$ и $g$.

Очевидно, что автомат $U$ в определении 3 задается неоднозначно. В данной работе более существенным фактором является не внутренняя структура самого автомата, а мощность множества состояний $\mathcal{N}$. В дальнейшем будем говорить, что $g$ соответствует конечный автомат, если $\mathcal{N}-$ конечное множество, или бесконечный автомат, если не существует соответствующего автомата с конечным числом состояний.

Определим ГПСЧ, порождаемый преобразованием $F_{c}(x)$, на множестве целых 2-адических чисел, полагая

$$
x_{i+1}=\mathbf{F}_{\mathbf{C}}\left(x_{i}\right)=x_{i}+\left(x_{i}^{2} \vee \mathbf{C}\right): \mathbf{Z}_{2} \rightarrow \mathbf{Z}_{2},
$$

где $\mathbf{C} \in \mathbf{Z}_{2}$ и $\mathbf{C} \equiv c\left(\bmod 2^{n}\right)$. В разделе 4 выявляются условия, при которых $\mathbf{F}_{\mathbf{C}}(x)$ соответствует конечному автомату (см. теорему 4) или бесконечному автомату (см. теоремы 5, 6).

В разделе 5 на основе данных о свойствах соответствующих автоматов рассматривается распределение пар

$$
\left(\frac{x \bmod p^{n}}{p^{n}}, \frac{\mathbf{F}_{\mathbf{C}}(x) \bmod p^{n}}{p^{n}}\right) \in[0,1]^{2}
$$

при всевозможных значениях $n \in \mathbf{N}$ и $x \in \mathbf{Z}_{2}$. Анализируются значения меры Лебега замыкания множества всех таких пар.

Рассматриваются свойства множества всех предельных неизолированных точек этого множества (раздел 6) и находится размерность Хаусдорфа (теорема 14) этого множества в случае, когда $\mathbf{C} \in \mathbf{Z}_{2} \cap \mathbf{Q}$.

\section{2. Известные свойства}

Важным свойством ГПСЧ является условие максимальности периода вырабатываемой гаммы из любого начального состояния. Функция $F_{c}(x)$ транзитивна по модулю $2^{n}$ для любого натурального $n$, то есть представляет собой перестановку с единственным циклом, если $c \equiv 5(\bmod 8)$ или $c \equiv 7(\bmod 8)$. Данное условие можно переписать с использованием $j$-й координатной функции:

$$
\delta_{0}(c)=\delta_{2}(c)=1,
$$

где

$$
\delta_{j}(x)=\frac{1}{2^{j}}\left(x-\left(x \bmod 2^{j}\right)\right) \quad(\bmod 2) .
$$


Отметим, что $\delta_{j}(x)$ является значением коэффициента при $2^{j}$ в двоичном разложении $x=\sum_{j=0}^{n-1} \delta_{j}(x) 2^{j}$.

Известно (см. [11]), что для $c<2^{n / 3}$ сложность определения внутреннего состояния генератора при условии, что на выход в качестве гаммы поступают значения $m=n / 2$ старших бит этого состояния, составляет $2^{n / 3}$ по времени и по памяти. При $c \geqslant 2^{n / 3}$ данная атака не применима.

В статье [7] рассматривается случай $c \leqslant 2^{n / 4}$. Получение внутреннего состояния генератора при тех же условиях имеет временную сложность $O\left(2^{n / 4}\right)$ и сложность по памяти $O\left(n \log _{2} n\right)$. Поэтому предлагается выбирать значения $c>2^{n / 4}$.

В работах $[16,17]$ рассматриваются свойства координатной функции и находится следующее линейное свойство:

$$
\delta_{j}\left(x_{i+2^{j-1}}\right)+\delta_{j}\left(x_{i}\right) \equiv \delta_{j-1}\left(x_{i}\right)+a_{2} \delta_{1}\left(x_{i}\right)+a_{1} \delta_{0}\left(x_{i}\right)+a_{0} \quad(\bmod 2),
$$

где $a_{i}-$ константы, зависящие от $c$.

В 2008 г. к похожему соотношению пришли Ванг и Ки (см. [18]) только в классе полиномов. При использовании данного соотношения сложность нахождения внутреннего состояния, как отмечают авторы, эквивалентна полному перебору.

\section{3. Распределение пар в $Z / 2^{n} \mathbf{Z}$}

Для анализа качества гаммы, вырабатываемой генераторами псевдослучайных чисел, помимо различных пакетов оценочных тестов (NIST, Diehard и другие) важны графические тесты, позволяющие находить скрытые зависимости. В некоторых случаях становится возможным описать предсказанные на графике зависимости, что в конечном счете приводит к пониманию внутренней структуры ГПСЧ и созданию эффективных отличительных тестов гаммы от истинно случайной последовательности. К группе графических тестов относится исследование свойств распределения пар на плоскости.

Генератор Климова-Шамира $F_{c}(x)$ обладает как квадратичными, так и линейными свойствами, в зависимости от выбранной константы $c$. Другими словами, точки $\left(x_{i_{1}}, F_{c}\left(x_{i_{1}}\right)\right),\left(x_{i_{2}}, F_{c}\left(x_{i_{2}}\right)\right),\left(x_{i_{3}}, F_{c}\left(x_{i_{3}}\right)\right), \ldots$ в некоторые моменты $i_{1}, i_{2}, i_{3}, \ldots$ ложатся на параболы или на прямые, наблюдаемые визуально на графике распределения на плоскости (в квадрате $\left.[0,1]^{2}\right)$. Каждой паре $\left(x_{i}, x_{i+1}\right) \in\left(\mathbf{Z} / 2^{n} \mathbf{Z}\right)^{2}$ ставится в соответствие точка на плоскости $\left(x_{i} / 2^{n}, x_{i+1} / 2^{n}\right) \in[0,1]^{2}$.

Далее рассматриваются некоторые свойства распределения, явно показывающие неудовлетворительные качества гаммы, вырабатываемой ГПСЧ Климова-Шамира, при всевозможных значениях $c \in \mathbf{Z} / 2^{n} \mathbf{Z}$, в особенности при больших значениях $c$, предложенных в вышеупомянутых работах.

\section{1. Разбиение на полосы}

Множество всевозможных значений пар $\left(x, F_{c}(x)\right)$ обозначим

$$
\Gamma_{c}=\left\{\left(x, F_{c}(x)\right): x \in \mathbf{Z} / 2^{n} \mathbf{Z}\right\} .
$$

Замечание 1. При с таких, что $\delta_{0}(c)=\delta_{2}(c)=1$, множество всевозможных значений пар $\left(x_{i-1}, x_{i}\right)$ элементов последовательности $x_{i}=F_{c}\left(x_{i-1}\right)$, порожденных из произвольного начального состояния $x_{0} \in \mathbf{Z} / 2^{n} \mathbf{Z}$, совпадает с

$$
\Gamma_{c}=\left\{\left(x_{i-1}, x_{i}\right): x_{0} \in \mathbf{Z} / 2^{n} \mathbf{Z}, x_{i}=F_{c}\left(x_{i-1}\right)\right\}
$$


Пусть

$$
c=\sum_{i=0}^{n-1} c_{i} 2^{i}, \quad c_{i} \in\{0,1\}
$$

Введем представление $c$ в виде двоичного вектора

$$
\begin{aligned}
& c=\left(\begin{array}{llllllll}
\ldots & 1, & 0, & \ldots, & 0, & 1, & \ldots, & 1
\end{array}\right)_{2} \\
& \ldots \quad \tau_{1}^{1}, \quad \tau_{0}^{0}+l_{0}^{0}-1, \quad \ldots, \quad \tau_{0}^{0}, \quad \tau_{0}^{1}+l_{0}^{1}-1, \quad \ldots, \quad \tau_{0}^{1}
\end{aligned}
$$

где под каждой компонентой записано число вида $\tau_{i}^{j}+t$, где $t \in\left\{0,1, \ldots, l_{i}^{j}-1\right\}$, $j \in\{0,1\}$ такое, что $c_{\tau_{i}^{j}+t}=j$. Другими словами в двоичной записи $c$ все коэффициенты с индексами от $\tau_{i}^{j}$ до $\tau_{i}^{j}+l_{i}^{j}-1$ равны $j$, а $i$ указывает, что данная подпоследовательность, состоящая только из нулей или только из единиц, является $i$-й подпоследовательностью соответствующего типа.

Пример 1. Например, $\tau_{0}^{1}=0, l_{0}^{1}=1, \tau_{1}^{1}=2, l_{1}^{1} \geqslant 1$ для всех $c \equiv 5(\bmod 8)$.

Обозначим через $k^{1}-1\left(k^{0}-1\right)$ номер последней подпоследовательности, состоящей из единиц (нулей), тогда

$$
c=\sum_{i=0}^{k^{1}-1}\left(\sum_{t=0}^{l_{i}^{1}-1} 2^{\tau_{i}^{1}+t}\right)=2^{n}-\sum_{i=0}^{k^{0}-1}\left(\sum_{t=0}^{l_{i}^{0}-1} 2^{\tau_{i}^{0}+t}\right) .
$$

Разобьем множество индексов $\{0,1, \ldots, n-1\}$ на два непересекающихся подмножества $\Lambda_{c}^{0} \cup \Lambda_{c}^{1}=\{0,1, \ldots, n-1\}$ :

$$
\Lambda_{c}^{j}=\bigcup_{i=0}^{k^{j}-1}\left\{\tau_{i}^{j}, \ldots, \tau_{i}^{j}+l_{i}^{j}-1\right\}, \quad j \in\{0,1\} .
$$

Определение 4. Будем называть $L_{\gamma}^{h}$ полосой ширины $h \in\left\{1,2, \ldots, 2^{n}-1\right\}$ и координатой $\gamma \in \mathbf{Z} / 2^{n} \mathbf{Z}$, если

$$
L_{\gamma}^{h}=\left\{(x, y): x, y \in \mathbf{Z} / 2^{n} \mathbf{Z}, \gamma+x \leqslant y \leqslant \gamma+x+h-1\right\} .
$$

Верна следующая теорема о разбиении на полосы.

Теорема 1. Для любого натурального $n \in \mathbf{N} u c \in\left\{1,2, \ldots, 2^{n}-1\right\}$

$$
\Gamma_{c} \subset \bigcup_{\gamma \in \operatorname{Coord}_{c}} L_{c+\gamma}^{2^{\tau_{0}^{1}}}
$$

где $\gamma \in \operatorname{Coord}_{c}$, если $\delta_{i}(\gamma)=0$ при

$$
i \in \begin{cases}\Lambda^{1}, & \tau_{0}^{1}>\tau_{0}^{0}, \\ \Lambda^{1} \cup\left\{\tau_{0}^{0}, \ldots, \tau_{0}^{0}+l_{0}^{0}-1\right\}, & \tau_{0}^{1}<\tau_{0}^{0} .\end{cases}
$$


Доказательство. Введем функцию

$$
G_{c}(x)=F_{c}(x)-x=x^{2} \vee c .
$$

Заметим, что $G_{c}(x) \geqslant c$ для всех $x$. Рассмотрим множество

$$
\Gamma_{c}^{\prime}=\left\{\left(x, G_{c}(x)\right): x \in \mathbf{Z} / 2^{n} \mathbf{Z}\right\} .
$$

Так как

$$
G_{c}(x)=x^{2} \vee c=c+\left(\bar{c} \wedge x^{2}\right)
$$

для $\Gamma_{c}^{\prime}$ верны включения

$$
\begin{aligned}
& \Gamma_{c}^{\prime} \subset\left\{(x, c+(\bar{c} \wedge \alpha)): x, \alpha \in \mathbf{Z} / 2^{n} \mathbf{Z}\right\}, \\
& \Gamma_{c}^{\prime} \subset \mathbf{Z} / 2^{n} \mathbf{Z} \times\left\{c+(\bar{c} \wedge \alpha): \alpha \in \mathbf{Z} / 2^{n} \mathbf{Z}\right\} .
\end{aligned}
$$

Рассмотрим множество

$$
\mathscr{A}_{c}=\left\{c+(\bar{c} \wedge \alpha): \alpha \in \mathbf{Z} / 2^{n} \mathbf{Z}\right\} .
$$

Случай 1: $\tau_{0}^{0}<\tau_{0}^{1}$. По построению $\tau_{0}^{0}=0, \tau_{0}^{0}+l_{0}^{0}=\tau_{0}^{1}$. Представим $\mathscr{A}_{c}$ как объединение подмножеств, у элементов $c+\gamma$ которых коэффициенты $\delta_{i}(\gamma)$ при значениях $i \in \Lambda_{c}^{0} \backslash\left\{\tau_{0}^{0}, \ldots, \tau_{0}^{0}+l_{0}^{0}-1\right\}$ произвольны, а при $i \in \Lambda_{c}^{1} \cup\left\{\tau_{0}^{0}, \ldots, \tau_{0}^{0}+l_{0}^{0}-1\right\}$ равны нулю:

$$
\mathscr{A}_{c}=\bigcup_{\gamma}\left\{c+\gamma, \ldots, c+\gamma+2^{\tau_{0}^{1}}-1\right\},
$$

где объединение взято по $\gamma$ таким, что $\delta_{i}(\gamma)=0$, если $i \in \Lambda_{c}^{1} \cup\left\{\tau_{1}^{0}, \ldots, \tau_{1}^{0}+l_{1}^{0}-1\right\}$.

Случай 2: $\tau_{0}^{0}>\tau_{0}^{1}$. Для данного случая $\tau_{0}^{1}=0, \tau_{0}^{1}+l_{0}^{1}=\tau_{0}^{0}$ и множество $\mathscr{A}_{c}$ можно представить как

$$
\mathscr{A}_{c}=\bigcup_{\gamma}\{c+\gamma\}
$$

где объединение взято по $\gamma$ таким, что $\delta_{i}(\gamma)=0$, если $i \in \Lambda_{c}^{1}$.

Объединяя оба случая (с учетом того, что при $\tau_{0}^{0}>\tau_{0}^{1}$ значение $2^{\tau_{0}^{1}}-1=0$ ) получим, что

$$
\left\{c+(\bar{c} \wedge \alpha): \alpha \in \mathbf{Z} / 2^{n} \mathbf{Z}\right\}=\bigcup_{\gamma \in \operatorname{Coord}_{c}}\left\{c+\gamma, \ldots, c+\gamma+2^{\tau_{0}^{1}}-1\right\} .
$$

Подчеркнем, что соответствие между $\Gamma_{c}$ и $\Gamma_{c}^{\prime}$ выражается следующим образом:

$$
\Gamma_{c}=\left\{(x, y):(x, y)=\left(x^{\prime}, x^{\prime}+y^{\prime}\right), \forall\left(x^{\prime}, y^{\prime}\right) \in \Gamma_{c}^{\prime}\right\} .
$$

Теперь можно выписать общее выражение для $\Gamma_{c}$ :

$$
\Gamma_{c} \subset \bigcup_{\gamma \in \operatorname{Coord}_{c}}\left\{(x, x+c+\gamma+j): x \in \mathbf{Z} / 2^{n} \mathbf{Z}, 0 \leqslant j \leqslant 2^{\tau_{0}^{1}}-1\right\} .
$$

Для завершения доказательства остается переписать данное включение с использованием определения полосы:

$$
\Gamma_{c} \subset \bigcup_{\gamma \in \operatorname{Coord}_{c}} L_{c+\gamma}^{2^{1}} .
$$

Замечание 2. В данном представлении для всех ненулевых $c$ есть один недостаток: в случае $c \equiv 5(\bmod 8)$ или $c \equiv 7(\bmod 8)$, при которых $F_{c}(x)$ имеет максимальный период, значение $\tau_{0}^{1}=0$ и визуально на графике $\Gamma_{c}$ отследить данные полосы практически невозможно. Поэтому можно предложить обобщенное разбиение на полосы. 


\section{2. Обобщенное разбиение на полосы}

Для $j \in\left\{0,1, \ldots, k^{1}-1\right\}$ множеством координат полос будем называть множество

$$
\operatorname{Coord}_{c}(j)=\left\{\gamma: \gamma=\sum_{i=\tau_{j}^{1}}^{n-1} d_{i} 2^{i} \in \mathbf{Z} / 2^{n} \mathbf{Z}, d_{i} \in\{0,1\}, i \in \Lambda_{c}^{1} \Rightarrow d_{i}=1\right\} .
$$

Верна следующая теорема об обобщенном разбиении на полосы.

Теорема 2. Пусть $c \in \mathbf{Z} / 2^{n} \mathbf{Z}$ таково, что $\delta_{0}(c)=\delta_{2}(c)=1$, тогда для любого $j \in\left\{0,1, \ldots, k^{1}-1\right\}$

$$
\Gamma_{c} \subset \bigcup_{\gamma \in \operatorname{Coord}_{c}(j)} L_{c+\gamma}^{2^{\tau_{j}^{1}}} .
$$

Следствие 1. Число полос в обобщенном разбиении $\Gamma_{c}$ при $j \in\left\{0,1, \ldots, k^{1}-1\right\}$ равно $2^{\sum_{t=j+1}^{k^{0}} l_{t}^{0}}$.

Доказательство. Утверждение следует из того, что число независимых компонент $d_{i}$ равно $\sum_{t=j+1}^{k^{0}} l_{t}^{0}$.

Таким образом, все точки $\left(x, F_{c}(x)\right)$ попадают на полосы заданной ширины. Теорема 1 дает инструмент для анализа выхода генератора, когда известно значение $c$ и требуется построить оракул для определения следующего выхода генератора, а также в более сложных случаях, когда значение $c$ неизвестно, а имеется выход генератора, по которому сначала определяется значение $c$, а затем строится оракул.

Приведенная теорема о разбиении на полосы показывает, что при достаточно больших значениях $c$ (к примеру, при $c>2^{n / 4}$, как предлагается в вышеупомянутых работах) распределение на плоскости будет обладать значительными изъянами. Следовательно, выбор таких значений $c$ ведет к получению заведомо неравномерно распределенной гаммы.

Пример 2. Выберем $n=15, c=(101000000000101)_{2}, j=2, \tau_{j}^{1}=12, l_{j+1}^{0}=1$, $l_{k^{0}}^{0}=l_{4}^{0}=1$. Число полос равно $2^{1+1}=4$ (см. рис. 1 ).

Несмотря на то, что последовательность при данном $c$ будет иметь максимальный период, множество пар значений $\left(x, F_{c}(x)\right)$ будет ложиться на полосы, показанные на рис. 1. Непосредственно из теоремы об обобщенном разбиении на полосы следует, что есть две полосы шириной $2^{n-2}=2^{13}$, причем в каждой полосе находятся еще по две полосы шириной уже $2^{n-4}=2^{11}$.

В случае, когда значение $c<2^{n / 4}$, в проекции распределения пар на единичный квадрат $[0,1]^{2}$ прослеживаются квадратичные зависимости, не исчезающие с ростом $n$.

\section{3. Семейство квадратичных структур $\Pi^{+}$}

Для произвольных $a, b, \varepsilon \in \mathbf{Z} / 2^{n} \mathbf{Z}$ квадратичной структурой назовем множество вида

$$
Q(a, b, \varepsilon)=\left\{(x, y): x \in(a-\varepsilon, a+\varepsilon) \cap \mathbf{Z} / 2^{n} \mathbf{Z}, y=F_{c}(x)+b\right\} ;
$$

они образуют семейство $\Pi^{+}$, состоящее из структур $Q(a, b, \varepsilon)$ при всевозможных фиксациях $a, b, \varepsilon$. 


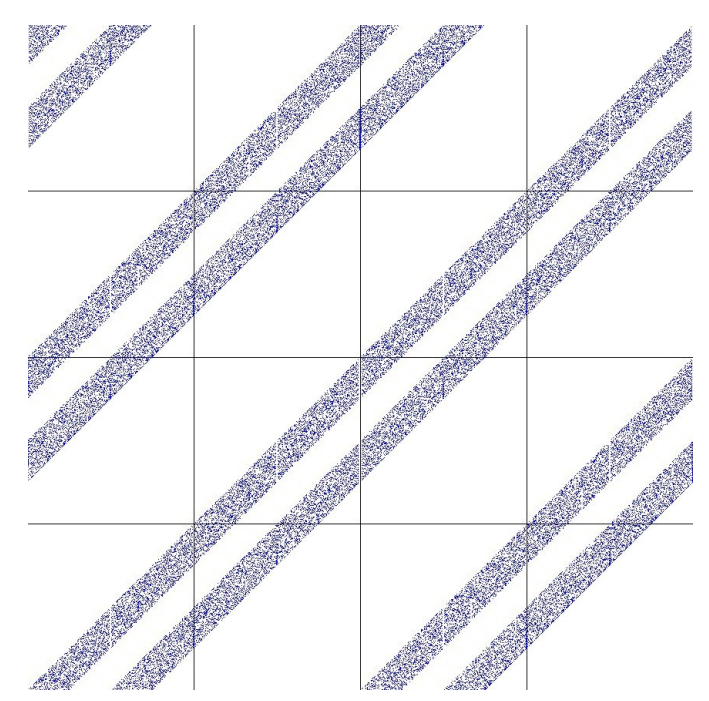

Puc. 1. $n=15, c=(101000000000101)_{2}$

Пусть $x=\alpha 2^{n-k}+\varepsilon$, где $\alpha \in \mathbf{Z} / 2^{k} \mathbf{Z}, \alpha \equiv 1(\bmod 2), \varepsilon \in \mathbf{Z} / 2^{\lfloor n / 4\rfloor} \mathbf{Z}, k \leqslant\lfloor n / 4\rfloor$, $c \leqslant 2^{n / 4}$. Тогда

$$
F_{c}(x)=\alpha 2^{n-k}+\varepsilon+\alpha 2^{n-k+1} \varepsilon+\left(\varepsilon^{2} \vee c\right) .
$$

Представим $\varepsilon$ как $\varepsilon\left(\tilde{\varepsilon}^{\prime}, \tilde{\varepsilon}^{\prime \prime}\right)=2^{k-1} \tilde{\varepsilon}^{\prime}+\tilde{\varepsilon}^{\prime \prime}$ :

$$
F_{c}(x)=\alpha 2^{n-k}+\varepsilon\left(\tilde{\varepsilon}^{\prime}, \tilde{\varepsilon}^{\prime \prime}\right)+\alpha 2^{n-k+1} \tilde{\varepsilon}^{\prime \prime}+\left(\varepsilon^{2}\left(\tilde{\varepsilon}^{\prime}, \tilde{\varepsilon}^{\prime \prime}\right) \vee c\right) .
$$

Фиксировав произвольное $\tilde{\varepsilon}^{\prime \prime} \in \mathbf{Z} / 2^{k-1} \mathbf{Z}$, получим равенство

$$
F_{c}\left(\alpha 2^{n-k}+\varepsilon\left(\tilde{\varepsilon}^{\prime}, \tilde{\varepsilon}^{\prime \prime}\right)\right)=F_{c}\left(\varepsilon\left(\tilde{\varepsilon}^{\prime}, \tilde{\varepsilon}^{\prime \prime}\right)\right)+\alpha 2^{n-k}+\alpha 2^{n-k+1} \tilde{\varepsilon}^{\prime \prime} .
$$

Таким образом, семейство квадратичных структур, параметризованных по $\alpha$ и $\tilde{\varepsilon}^{\prime \prime}$, представимо в виде

$$
\Pi^{+}=\left\{Q\left(\alpha 2^{n-k}+\tilde{\varepsilon}^{\prime \prime}, \alpha 2^{n-k}+\alpha 2^{n-k+1} \tilde{\varepsilon}^{\prime \prime}, 2^{k-1} \tilde{\varepsilon}^{\prime}\right)\right\}
$$

Пример 3. Пусть $n=16, c=5=(101)_{2}, k=1, y, \tilde{\varepsilon}^{\prime \prime} \in\{0,1\}$, тогда семейство $\Pi^{+}$ содержит 7 квадратичных структур такого вида, две из которых можно явно проследить на рис. 2, что показывает наличие нелинейных зависимостей в распределении пар даже при малых значениях $c$.

\section{4. Семейство парабол $\Pi^{-}$}

Введем семейство парабол

$$
\Pi^{-}=\left\{Q_{i}^{t}(x): i \in \mathbf{Z} / 2^{n-t+1} \mathbf{Z}, t \in \mathbf{Z} / 2^{n} \mathbf{Z}\right\},
$$

где

$$
Q_{i}^{t}(x)=-\frac{1}{2^{t}}\left(x-i 2^{t-1}\right)^{2}+i(i-1) 2^{t-1}+2^{t-2}+c .
$$




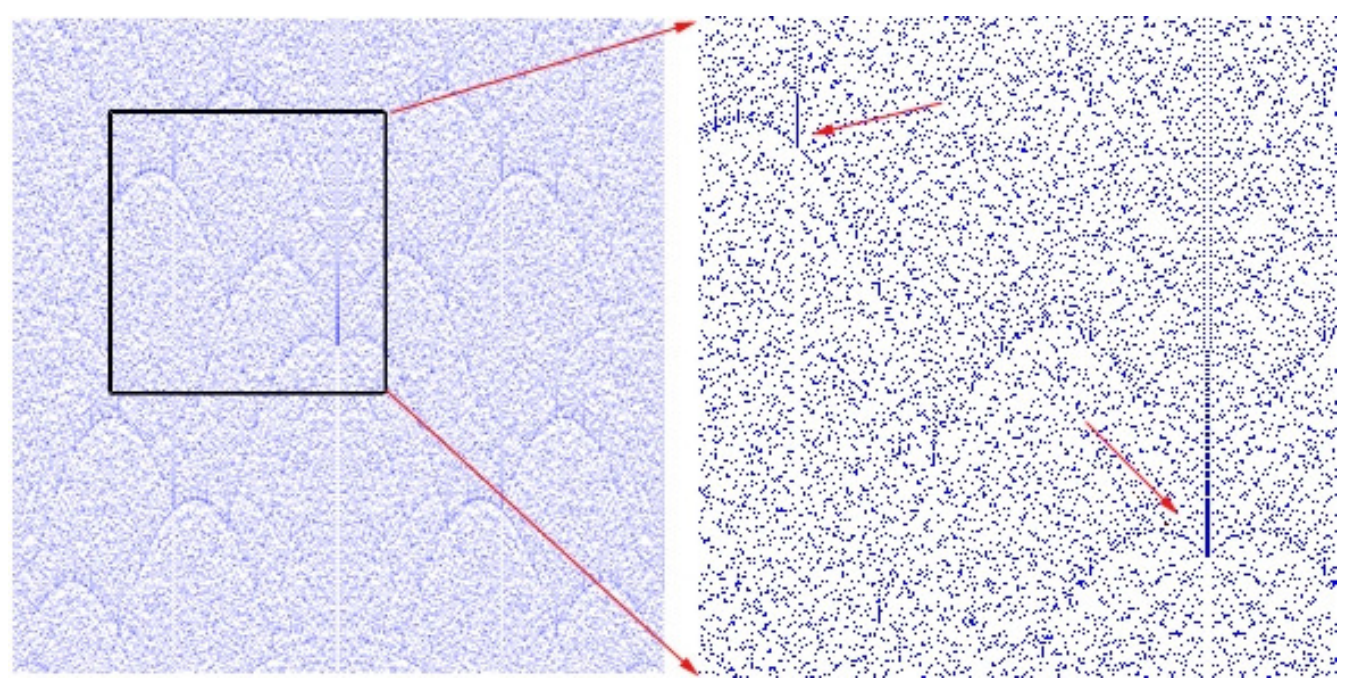

Pис. 2. $n=16, c=(101)_{2}$

Рассмотрим произвольный элемент семейства $\Pi^{-}$и найдем множество точек, где $Q_{i}^{t}(x)=F_{c}(x)$, для некоторых фиксированных значений $i, t$. Предварительно наложим небольшие ограничения на $x$, именно, пусть $x^{2} \vee c=x^{2}+c$ и $c \in \mathbf{Z} / 2^{\lfloor n / 4\rfloor} \mathbf{Z}$. Тогда

$$
\begin{aligned}
-\frac{1}{2^{t}}\left(x-i 2^{t-1}\right)^{2}+i(i-1) 2^{t-1}+2^{t-2}+c & =x^{2}+x+c-\left(1+2^{-t}\right) x^{2}+(i-1) x \\
& =-i^{2} 2^{t-2}+i^{2} 2^{t-1}-i 2^{t-1}+2^{t-2} \\
& =0, \\
-\left(1+2^{t}\right) x^{2}+(i-1) 2^{t} x+\left((i-1) 2^{t-1}\right)^{2} & =0 .
\end{aligned}
$$

Пусть $t-1 \geqslant n / 2$, тогда $x=2^{n / 2} \tilde{x}$ будет являться решением уравнения $Q_{i}^{t}(x)=F_{c}(x)$ для всех $\tilde{x} \in \mathbf{Z} 2^{\lceil n / 2\rceil} \mathbf{Z}$. Таким образом, верно следующее утверждение.

Предложение 1. Пусть $c \in \mathbf{Z} 2^{\lfloor n / 4\rfloor} \mathbf{Z}$, тогда число точек $x \in \mathbf{Z} / 2^{n} \mathbf{Z}$ таких, что $F_{c}(x) \in \Pi^{-}$, не меньше $2^{\lceil n / 2\rceil}$.

Пример 4. Пусть $t=n-3, i \in \mathbf{Z} 2^{4} \mathbf{Z}, n=16, c=5$. Данные параболы явно видны на рис. 3 .

\section{5. Определение значения $c$}

Определение внутреннего состояния генератора при неизвестном значении $c$ в случае наличия нескольких пар последовательных выходов гаммы $\left(x_{i_{1}}, x_{i_{1}+1}\right),\left(x_{i_{2}}, x_{i_{2}+1}\right), \ldots$ требует наличия малого количества таких пар.

Рассмотрим 0-й бит $x_{i+1}=F_{c}\left(x_{i}\right)$ :

$$
\delta_{0}\left(x_{i+1}\right) \equiv \delta_{0}\left(x_{i}\right)+\left(\delta_{0}\left(x_{i}^{2}\right) \vee \delta_{0}(c)\right)(\bmod 2)
$$

Если $\delta_{0}\left(x_{i}\right)=0$, то $\delta_{0}(c)$ определяется однозначно, если же $\delta_{0}\left(x_{i}\right)=1$, то $\delta_{0}\left(x_{i+1}\right)=0$ или потребуется взять другую пару. Пусть определены младшие $s-1$ бит $c$. Рассмотрим 


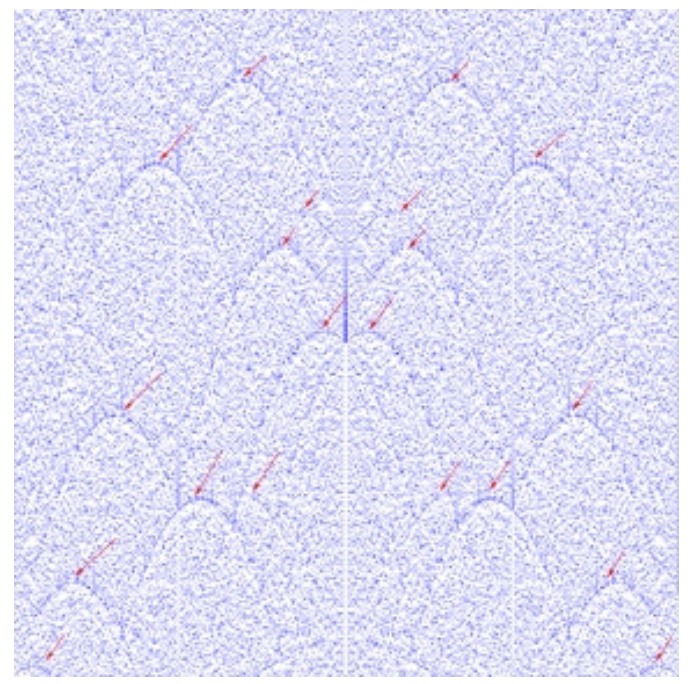

Puc. 3. $n=16, c=(101)_{2}$

бит

$$
\delta_{s}\left(x_{i+1}\right) \equiv \delta_{s}\left(x_{i}\right)+\left(\delta_{s}\left(x_{i}^{2}\right) \vee \delta_{s}(c)\right)+\delta_{s}\left(r_{i}\right)(\bmod 2),
$$

где $\delta_{s}\left(r_{i}\right)$ - бит переноса разряда. Считая, что величины $\delta_{s}\left(x_{i+1}\right), \delta_{s}\left(x_{i}\right), \delta_{s}\left(x_{i}^{2}\right), \delta_{s}\left(r_{i}\right)$ могут принимать произвольные значения из $\{0,1\}$, получаем, что из 16 возможных состояний допустимы 13, из которых только в 4 есть неоднозначность в определении $\delta_{s}(c)$.

Следовательно, с вероятностью 4/13 невозможно однозначно определить $\delta_{s}(c)$. Если имеется $t$ таких пар, то с вероятностью $(4 / 13)^{t}$ невозможно будет определить $\delta_{s}(c)$ ни на одной из пар.

Теорема 3. Если имеется $t$ различных пар $\left(x_{i}, F_{c}\left(x_{i}\right)\right) \in\left(\mathbf{Z} / 2^{n} \mathbf{Z}\right)^{2}, i \in\{0,1, \ldots, t-1\}$, то значение с можно определить с вероятностью $P \geqslant 1-n(4 / 13)^{t}$.

Следствие 2. При $n=32$ для определения значения константы $c$ с вероятностью $P \geqslant 0,999$ требуется не более 9 различных пар $\left(x_{i}, F_{c}\left(x_{i}\right)\right)$.

\section{6. Линейная зависимость координатной функции}

Как было упомянуто во введении, в работах $[16,17]$ находится следующая линейная зависимость в $j$-й координатной функции:

$$
\delta_{j}\left(x_{i+2^{j-1}}\right)+\delta_{j}\left(x_{i}\right) \equiv \delta_{j-1}\left(x_{i}\right)+a_{2} \delta_{1}\left(x_{i}\right)+a_{1} \delta_{0}\left(x_{i}\right)+a_{0} \quad(\bmod 2),
$$

где $a_{i}-$ константы, зависящие от $c$. Отметим, что для всех совместимых преобразований максимального периода верно соотношение

$$
\delta_{j}\left(x_{i}\right)+\delta_{j}\left(x_{i+2^{j}}\right) \equiv 1(\bmod 2) .
$$

Отсюда несложно показать, что в общем случае $\delta_{j}\left(x_{i}\right)+\delta_{j}\left(x_{i+2^{j-1}}\right)$ является функцией от переменных $\delta_{j-1}\left(x_{i}\right), \ldots, \delta_{0}\left(x_{i}\right)$, причем от $\delta_{j-1}\left(x_{i}\right)$ она зависит линейно. 


\section{4. Оценка числа состояний автоматов}

Т-функции могут быть рассмотрены как автоматные функции (см. гл. 8 в [6]), соответствующие автоматам Мили, и их свойства существенным образом зависят от свойств этих автоматов, в частности, от того, является автомат конечным или бесконечным. Напомним, что из-за неоднозначности задания автомата будем говорить, что Т-функции соответствует бесконечный автомат, если не существует автомата с конечным числом состояний. Далее будет показано, что $\mathbf{F}_{\mathbf{C}}(x)$ может соответствовать как конечный, так и бесконечный автомат.

Любая функция $f: \mathbf{Z}_{p} \rightarrow \mathbf{Z}_{p}$, удовлетворяющая условию Липшица с константой 1 , для любого натурального $k \in \mathbf{N}$ может быть представлена в виде

$$
f(x)=f\left(x \bmod p^{k}\right)+p^{k} g_{z}(y),
$$

где

$$
y=\frac{1}{p^{k}}\left(x-\left(x \bmod p^{k}\right)\right) \in \mathbf{Z}_{p}, \quad z=z(x)=x \bmod p^{k},
$$

a $g_{z}: \mathbf{Z}_{p} \rightarrow \mathbf{Z}_{p}$ также удовлетворяют условию Липшица с константой 1.

В случае, если $f$ соответствует некоторому автомату с конечным числом состояний, то число различных функций $g_{z}$ при всевозможных значениях $z=x \bmod p^{k}$ и всех $k \in \mathbf{N}$ также конечно. Следовательно, существует такое натуральное $N \in \mathbf{N}$, что $g_{z}$ для любого $k>N$ при $z=x \bmod p^{k}$ принадлежит

$$
G_{N}=\left\{g_{z}: z=x \bmod p^{N}\right\}
$$

Таким образом, всего различных функций $g_{z}$ не больше чем $p^{N}$.

Если $f$ соответствует автомату с бесконечным числом состояний, то для любого натурального $N \in \mathbf{N}$ существует такое натуральное $k>N$, что $g_{z}$ при $z=x \bmod p^{k}$ не принадлежит $G_{N}$.

Теорема 4. Пусть $\mathbf{C} \in \mathbf{Z}_{2}$ является отрицательным ичельм числом, тогда $\mathbf{F}_{\mathbf{C}}$ соответствует конечному автомату.

Доказательство. Число $\mathbf{C}$ может быть представлено в виде $\mathbf{C}=e^{-} 2^{k}+c$, где $c \in \mathbf{Z} / 2^{k} \mathbf{Z}$, а $e^{-}=(\ldots 111111)_{2}=-1 \in \mathbf{Z}_{2}$. Тогда

$$
\mathbf{F}_{\mathbf{C}}(x)=x+\left(x^{2} \vee\left(e^{-} 2^{k}+c\right)\right)=x+e^{-} 2^{k}+\left(\left(x^{2} \bmod 2^{k}\right) \vee c\right),
$$

так как $e^{-} \vee x=(\ldots 111111)_{2} \vee x=(\ldots 111111)_{2}=e^{-}$. Пусть $z=x \bmod 2^{k}$, тогда

$$
\begin{aligned}
\mathbf{F}_{\mathbf{C}}(x) & =\mathbf{F}_{\mathbf{C}}\left(x \bmod 2^{k}\right)+2^{k} g_{z}(y) \\
& =\left(x \bmod 2^{k}\right)+\left(\left(x^{2} \bmod 2^{k}\right) \vee c\right)+\left(y+e^{-}\right) 2^{k} .
\end{aligned}
$$

Следовательно,

$$
g_{z}(y)=y+e^{-}=y-1 .
$$

Таким образом, для всех натуральных $N>k$ мощность $\left|G_{N}\right|=\left|G_{k}\right|$ и $\mathbf{F}_{\mathbf{C}}$ представима конечным автоматом. 
Другими словами, распределение на плоскости при отрицательном рациональном целом $\mathbf{C}$ будет соответствовать распределению функции $f(x)=x-1$, что и было показано на примере и рисунках в [6].

Теорема 5. Пусть $\mathbf{C} \in \mathbf{Z}_{2}$ является положительным цельмм числом, тогда $\mathbf{F}_{\mathbf{C}}$ не представим конечным автоматом.

Доказательство. Величина $\mathbf{C}$ может быть представлена в виде $\mathbf{C}=c$, где $c \in \mathbf{Z} / 2^{k} \mathbf{Z}$. Тогда

$$
\mathbf{F}_{\mathbf{C}}(x)=x+\left(x^{2} \vee c\right)=\left(x \bmod 2^{k}\right)+y 2^{k}+\left(\left(x \bmod 2^{k}+y 2^{k}\right)^{2} \vee c\right) .
$$

Так как

$$
\begin{aligned}
\left(x \bmod 2^{k}+y 2^{k}\right)^{2} \vee c & =y^{2} 2^{2 k}+2\left(x \bmod 2^{k}\right) y 2^{k}+\left(\left(x \bmod 2^{k}\right)^{2} \vee c\right) \\
\mathbf{F}_{c}\left(x \bmod 2^{k}\right) & =\left(x \bmod 2^{k}\right)+\left(\left(x \bmod 2^{k}\right)^{2} \vee c\right),
\end{aligned}
$$

получаем

$$
\begin{aligned}
\mathbf{F}_{\mathbf{C}}(x) & =\left(y^{2} 2^{k}+2\left(x \bmod 2^{k}\right) y+y\right) 2^{k}+F_{c}\left(x \bmod 2^{k}\right) \\
& =\left(y^{2} 2^{k}+2 z y+y\right) 2^{k}+F_{c}(z) \\
& =q_{z}(y) 2^{k}+F_{c}(z),
\end{aligned}
$$

где

$$
q_{z}(y)=y^{2} 2^{k}+2 z y+y
$$

есть полином второй степени. Согласно [6], если автоматная функция $\Psi=\Psi_{\imath}$ является полиномом степени, большей 1 , то соответствующий ей автомат $U$ не имеет эквивалентных состояний и, соответственно, бесконечен.

В случае, если существует конечный автомат, реализующий $\mathbf{F}_{\mathbf{C}}$, то можно также построить и конечный автомат, реализующий полином второй степени, что приводит к противоречию.

Теорема 6. Пусть $\mathbf{C} \in \mathbf{Z}_{2} \backslash \mathbf{Z}$, тогда любой автомат Мили $И$, соответствуюший $\mathbf{F}_{\mathbf{C}}$, не имеет эквивалентных состояний.

Доказательство. Предположим противное, то есть, пусть существует такой автомат $U$, соответствующий $\mathbf{F}_{\mathbf{C}}$, что при двух различных входах конечной длины $U$ перейдет в эквивалентные состояния, при которых для любой входной последовательности выход будет одинаковым в любой момент времени. Иными словами существуют такие $x \in \mathbf{Z} / 2^{l} \mathbf{Z}$ и $y \in \mathbf{Z} / 2^{s} \mathbf{Z}, l, s \in \mathbf{N}$, что

$$
\delta_{t+l}\left(\mathbf{F}_{\mathbf{C}}\left(x+z 2^{l}\right)\right)=\delta_{t+s}\left(\mathbf{F}_{\mathbf{C}}\left(y+z 2^{s}\right)\right)=\gamma_{t}(z)
$$

для всех $z \in \mathbf{Z}_{2}$ и $t \in \mathbf{N} \cup\{0\}$,

$$
\begin{aligned}
\gamma_{t}(z) & \left.=\gamma_{t}(z, x, y)=\mathbf{F}_{\mathbf{C}}\left(x+z 2^{l}\right)\right) \\
& =x+z 2^{l}+\left(\left(x^{2}+z x 2^{l+1}+z^{2} 2^{2 l}\right) \vee \mathbf{C}\right) .
\end{aligned}
$$


Пусть $t>2 l$, тогда

$$
\begin{aligned}
\gamma_{t}(z) & =\delta_{t+l}\left(\mathbf{F}_{\mathbf{C}}\left(x+z 2^{l}\right)\right) \\
& =\delta_{t}(z) \oplus\left(\delta_{t}\left(2 z x+z^{2} 2^{l}\right) \vee \delta_{t+l}(\mathbf{C})\right) \oplus r_{t}^{x}(z),
\end{aligned}
$$

где $r_{t}^{x}(z)$ - мажоритарная функция, то есть функция вида $m(x, y, z)=x y \vee x z \vee y z$, именно,

$$
\begin{aligned}
r_{t}^{x}(z) & =m\left(\delta_{t-1}\left(2 z x+z^{2} 2^{l}\right) \vee \delta_{t+l-1}(\mathbf{C}), \delta_{t-1}(z), r_{t-1}^{x}\right) \\
& =m\left(\gamma_{t-1}(z), \delta_{t-1}(z), r_{t-1}^{x}\right) .
\end{aligned}
$$

Аналогичные соотношения верны и для $r_{t}^{y}(z)$ при $t>2 s$.

Положим

$$
r_{0}^{x}(z)=r_{0}^{y}(z) \oplus \tau, \quad \tau \in\{0,1\},
$$

тогда для всех $t \in \mathbf{N}$

$$
r_{t}^{x}(z)=r_{t}^{y}(z) \oplus \tau .
$$

При $t>4 \max (l, s)+1$ таком, что

$$
\delta_{t+l}(\mathbf{C})=0, \quad \delta_{t+s}(\mathbf{C})=1,
$$

для всех $z \in \mathbf{Z}_{2}$ должно быть истинно выражение

$$
\begin{aligned}
\delta_{t}\left(2 z x+z^{2} 2^{l}\right) \vee \delta_{t+l}(\mathbf{C}) & =\delta_{t}\left(2 z x+z^{2} 2^{l}\right) \\
& =\gamma_{t}(z) \oplus \delta_{t}(z) \oplus r_{t}^{x} \oplus r_{t}^{y} \\
& =\delta_{t}\left(2 z y+z^{2} 2^{s}\right) \vee \delta_{t+s}(\mathbf{C}) \oplus \tau \\
& =1 \oplus \tau .
\end{aligned}
$$

Пусть $z=2^{t-1}$, тогда

$$
\delta_{t}\left(2 z x+z^{2} 2^{l}\right)=\delta_{t}\left(2^{t} x+2^{l+2(t-1)}\right)=\delta_{t}\left(2^{t} x\right)=\delta_{0}(x)=1 .
$$

Далее положим

$$
\begin{gathered}
z=2^{t-1}+2^{t-1-\xi}, \quad \xi \in\{1, \ldots, l-1\}, \\
\delta_{t}\left(2^{t} x+2^{t-\xi} x+\left(2^{2(t-1)}+2^{2(t-1-\xi)}+2^{(t-1)+(t-1-\xi)+1}\right) 2^{l}\right)=\delta_{0}(x)+\delta_{\xi}(x)=1 .
\end{gathered}
$$

Следовательно, $x \equiv 1\left(\bmod 2^{l}\right)$ при условии (3). Рассмотрим два состояния $x^{\prime}=x+\left(z \bmod 2^{\zeta}\right) 2^{l}$ и $y^{\prime}=y+\left(z \bmod 2^{\zeta}\right) 2^{s}$ при $\zeta \in\left\{1,2, \ldots, 2^{\min (l, s)}\right)$. В этом случае значение $\gamma_{t}\left(z^{\prime}, x^{\prime}, y^{\prime}\right)$ также представимо в виде (1), так как $t$ было выбрано таким образом, что $t>4, \max (l, s)+1>2(\max (l, s)+\min (l, s))$, что приводит к соответствующему ограничению на $x^{\prime}$ :

$$
x^{\prime}=x+\left(z \bmod 2^{\zeta}\right) 2^{l} \equiv 1 \quad\left(\bmod 2^{l+\min (l, s)}\right) .
$$

Отсюда получаем, что $z \bmod 2^{\zeta}=0$ и предположение о существовании двух эквивалентных состояний автомата $U$ при условии (3) неверно.

В случае, когда $\delta_{t+l}(\mathbf{C})=\delta_{t+s}(\mathbf{C})$ для всех $t$, достаточно взять $t>4 \max (l, s)+1$ такое, при котором $\delta_{t+l}(\mathbf{C})=\delta_{t+s}(\mathbf{C})=0$, так как по условию $\mathbf{C} \in \mathbf{Z}_{2} \backslash \mathbf{Z}$ и, следовательно, для 
любого натурального $n$ найдется $\delta_{n+j}(\mathbf{C})=0$. Соответственно, для всех $z \in \mathbf{Z}_{2}$ должно выполняться равенство $\delta_{t}\left(2 z x+z^{2} 2^{l}\right)=\delta_{t}\left(2 z y+z^{2} 2^{s}\right)$, из чего по аналогии, подставляя значения $z=2^{t-1}+2^{t-1-\xi}$ при различных $\xi$, получим, что $x \equiv y\left(\bmod 2^{\min (l, s)}\right)$.

Таким образом, не существует автомата $U$, соответствующего $\mathbf{F}_{\mathbf{C}}$ и имеющего эквивалентные состояния.

Следствие 3. Пусть $\mathbf{C} \in \mathbf{Z}_{2} \backslash \mathbf{Z}$, тогда $\mathbf{F}_{\mathbf{C}}$ не представим конечным автоматом.

\section{5. Распределение пар в $Z_{2}$}

Для оценки распределения пар $\left(x_{i}, \mathbf{F}_{\mathbf{C}}\left(x_{i}\right)\right)$ в $\mathbf{Z}_{2}$ используется техника, предложенная в [6].

Для заданного преобразования $f: \mathbf{Z}_{p} \rightarrow \mathbf{Z}_{p}$, фиксированных $k, n \in \mathbf{N}$, рассмотрим множество

$$
\mathscr{P}_{n}^{k}(f)=\left\{\left(\frac{x}{p^{n}}, \frac{f(x) \bmod p^{n}}{p^{n}}, \ldots, \frac{f^{k-1}(x) \bmod p^{n}}{p^{n}}\right): x \in \mathbf{Z} / p^{n} \mathbf{Z}\right\}
$$

и замыкание объединения $\mathscr{P}_{n}^{k}(f)$ при всех $n$

$$
\mathscr{P}^{k}(f)=\mathrm{Cl}\left(\bigcup_{n=1}^{\infty} \mathscr{P}_{n}^{k}(f)\right)
$$

где $\mathrm{Cl}(A)$ является замыканием ограниченного множества $A \subset[0,1]^{2}$. Следовательно, $\mathscr{P}^{k}(f)$ измеримо по Лебегу.

Определение 5. Введем

$$
\alpha_{k}(f)=\zeta_{k}\left(\mathscr{P}^{k}(f)\right)
$$

где $\zeta_{k}$ - мера Лебега на $\mathbf{R}$.

Теорема 7. Если $f: \mathbf{Z}_{p} \rightarrow \mathbf{Z}_{p}$ удовлетворяет условию Липшица с константой 1 , то $\alpha_{2}(f)$ равна 0 или 1 .

Эта теорема доказана в [6] и названа там законом нуля и единицы.

Пусть $\alpha_{2}(f)=1$, тогда для каждой точки $(a, b) \in[0,1]^{2}$, для произвольного вещественного $\varepsilon>0$ существует точка $(\tilde{u}, \tilde{v}) \in \bigcup_{n=1}^{\infty} \mathscr{P}_{n}^{k}(f)$, лежащая в $\varepsilon$-окрестности $U_{\varepsilon}(a, b)$ точки $(a, b):(\tilde{u}, \tilde{v}) \in \mathscr{P}^{k}(f) \cap U_{\varepsilon}(a, b)$. По построению $\mathscr{P}^{k}(f)$, для $(\tilde{u}, \tilde{v})$ существует такое натуральное $t$, что значения $\tilde{u} \cdot p^{t}$ и $\tilde{v} \cdot p^{t}$ являются натуральными числами, а при $t-1-$ нет. Следовательно,

$$
f\left(\tilde{u} \cdot p^{t}\right) \equiv \tilde{v} \cdot p^{t} \quad\left(\bmod p^{t}\right) .
$$

В случае $\alpha_{2}(f)=0$ в любой $\varepsilon$-окрестности любой точки $(a, b) \in[0,1]^{2}$ найдется меньшая окрестность $U_{\mathcal{\varepsilon}^{\prime}}\left(a+\xi_{a}, b+\xi_{b}\right) \in U_{\varepsilon}(a, b)$, не имеющая общих точек с $\mathscr{P}^{k}(f)$ :

$$
U_{\varepsilon^{\prime}}\left(a+\xi_{a}, b+\xi_{b}\right) \cap \mathscr{P}^{k}(f)=\varnothing .
$$

Рассмотрим $\alpha_{2}\left(\mathbf{F}_{\mathbf{C}}\right)$ и покажем, что даже если $\mathbf{F}_{\mathbf{C}}$ соответствует бесконечному автомату, мера Лебега замыкания множества всех достижимых точек может быть равна как 0 , так и 1. 
Теорема 8. Если $\mathbf{C} \in \mathbf{Z}_{2}$ является отрицательным цуельцм числом, то $\alpha_{2}\left(\mathbf{F}_{\mathbf{C}}\right)=0$.

Доказательство. Так как при отрицательном целом значении $\mathbf{C}$ автоматная функция $\mathbf{F}_{\mathbf{C}}$ соответствует конечному автомату $(4), \alpha_{2}\left(\mathbf{F}_{\mathbf{C}}\right)=0$ по теореме 11.10 в [6].

Теорема 9. Если $\mathbf{C} \in \mathbf{Z}_{2}$ является положительным цельмм числом, то $\alpha_{2}\left(\mathbf{F}_{\mathbf{C}}\right)=1$.

Доказательство. Так как при положительном целом значении $\mathbf{C}$ автоматная функция $\mathbf{F}_{\mathbf{C}}$ соответствует бесконечному автомату $U$ (4) и $U$ может быть представлен как композиция некоторого конечного автомата, обрабатывающего конечное число начальных входных символов, и бесконечного автомата, соответствующего квадратичной функции $q_{z}(y)=y^{2} 2^{k}+2 z y+y$, по теореме 11.11 из [6] множество $\mathscr{P}^{2}(f)$ является всюду плотным на $[0,1]^{2}$ и, следовательно, $\alpha_{2}\left(\mathbf{F}_{\mathbf{C}}\right)=1$.

Определение 6. Пусть $x \in \mathbf{Z}_{p}$, тогда для всех натуральных $k, n \in \mathbf{N}$ определим функцию

$$
H_{p}(x, n, k)=\frac{1}{p^{n}}\left(\left(x \bmod p^{n+k}\right)-\left(x \bmod p^{n}\right)\right) .
$$

Ясно, что для любых допустимых значений аргументов $H_{p}(x, n, k) \in\left\{0,1, \ldots, p^{k}-1\right\}$.

Лемма 1. Пусть $f_{s}(x)=\left(x 2^{l}+s\right)^{2}: \mathbf{Z}_{2} \rightarrow \mathbf{Z}_{2}$, где $l \in \mathbf{N} \cup\{0\}, s \in\left\{0,1, \ldots, 2^{l}-1\right\}$. Тогда для любого $N \in \mathbf{N}$ и произвольных значений $a, b \in\left\{0,1, \ldots, 2^{N}-1\right\}$ для произвольного натурального $n>3(3 N+l+2)$ существует такое $x \in \mathbf{Z} / 2^{n} \mathbf{Z}$, что

$$
\begin{aligned}
H_{2}(x, n-N, N) \equiv a & \left(\bmod 2^{N}\right), \\
H_{2}\left(f_{s}(x), n+l-N, N\right) \equiv b & \left(\bmod 2^{N}\right) .
\end{aligned}
$$

Доказательство. Положим

$$
\xi=3 N+l+2 .
$$

Рассмотрим значение функции $f_{s}(x)$ при $x=a 2^{n-N}+x_{1} 2^{n-l-\xi}+2^{\xi-N-1}$, где $x_{1} \in\left\{0,1, \ldots, 2^{N}-1\right\}$ :

$$
\begin{aligned}
f_{s}(x)=\left(x 2^{l}+s\right)^{2}= & \left(a 2^{n-N+l}+x_{1} 2^{n-\xi}+2^{\xi-1-N+l}+s\right)^{2} \\
= & a^{2} 2^{n+N+(n-3 N+2 l)}+x_{1}^{2} 2^{n+N+(n-2 \xi-N))}+2^{2(\xi-1-N+l)} \\
& \quad+s^{2}+a x_{1} 2^{n+N+(n-\xi-2 N+l+1)}+a 2^{n+N+(\xi-3 N+2 l)} \\
& \quad+a s 2^{n+1-N+l}+x_{1} 2^{n-N+l}+x_{1} s 2^{n-\xi+1}+s 2^{\xi} .
\end{aligned}
$$

Так как

$$
\begin{gathered}
2^{2(\xi-1-N+l)}+s^{2}+s 2^{\xi}<2^{2 \xi}<2^{n-\xi}<2^{n-N+l-1}, \\
x_{1} s 2^{n-\xi+1}<2^{n-\xi+1+N+l}<2^{n-N-1},
\end{gathered}
$$

получаем, что

$$
H_{2}\left(2^{2(\xi-1-N+l)}+s^{2}+s 2^{\xi}+x_{1} s 2^{n-\xi+1}, n+l-N, N\right)=0 .
$$

3 Дискретная математика, т.23 №1 
Далее, $n-3 N+2 l>0, n-2 \xi-N>0, n-\xi-2 N+l+1>0, \xi-3 N+2 l>0$. Следовательно,

$$
\begin{aligned}
a^{2} 2^{n+N+(n-3 N+2 l)}+ & x_{1}^{2} 2^{n+N+(n-2 \xi-N))} \\
& +a x_{1} 2^{n+N+(n-\xi-2 N+l+1)}+a 2^{n+N+(\xi-2 N+l)} \equiv 0 \quad\left(\bmod 2^{n+N}\right)
\end{aligned}
$$

Таким образом,

$$
\begin{aligned}
H_{2}\left(f_{s}(x), n+l-N, N\right) & =H_{2}\left(a s 2^{n+1-N+l}+x_{1} 2^{n}, n+l-N, N\right) \\
& =H_{2}\left(2 a s+x_{1}, 0, N\right) .
\end{aligned}
$$

Условие (4) выполняется при значении $x_{1} \equiv b-2 a s\left(\bmod 2^{N}\right)$.

Теорема 10. Пусть $f_{s}(x)=\left(x 2^{l}+s\right)^{2}: \mathbf{Z}_{2} \rightarrow \mathbf{Z}_{2}$, где $l \in \mathbf{N} \cup\{0\}, s \in\left\{0,1, \ldots, 2^{l}-1\right\}$. Тогда $\alpha_{2}\left(f_{s}\right)=1$ для всевозможных значений параметров l $\mathrm{u} s$.

Доказательство. Достаточно доказать (см. замечание 11.12 в [6]), что для любого $N \in \mathbf{N}$ и произвольных значений $a, b \in\left\{0,1, \ldots, 2^{N}-1\right\}$ при некотором натуральном $n \geqslant N$ существует такое $x \in \mathbf{Z} / 2^{n} \mathbf{Z}$, что выполняется условие (4). По лемме 1 , при $n>3(3 N+l+2)$ такое $x$ существует.

Лемма 2. Пусть $f(x): \mathbf{Z}_{2} \rightarrow \mathbf{Z}_{2}, g(x)=x+f(x)$. Положим

$$
f_{s}(x)=f\left(x 2^{l}+s\right): \mathbf{Z}_{2} \rightarrow \mathbf{Z}_{2},
$$

где $l \in \mathbf{N} \cup\{0\}, s \in\left\{0,1, \ldots, 2^{l}-1\right\}$. Если $\alpha_{2}\left(f_{s}\right)=1$ при некоторых значениях параметров $l u s, m o \alpha_{2}\left(g_{s}\right)=1$.

Доказательство. Так как $\alpha_{2}\left(f_{s}\right)=1$, для любого $N \in \mathbf{N}$ и произвольных значений $a, b \in\left\{0,1, \ldots, 2^{N}-1\right\}$ существует такое натуральное $n>N$ и $x \in \mathbf{Z} / 2^{n} \mathbf{Z}$, что

$$
\begin{aligned}
H_{2}(x, n-N, N) & \equiv a \quad\left(\bmod 2^{N}\right), \\
H_{2}\left(f_{s}(x), n+l-N, N\right) & \equiv b-a \quad\left(\bmod 2^{N}\right) .
\end{aligned}
$$

Рассмотрим значение функции $g_{s}(x)$ при тех же значениях параметров:

$$
\begin{aligned}
g_{s}(x) & =f_{s}(x)+\left(x 2^{l}+s\right) \equiv(b-a) 2^{n}+\left(f_{s}(x) \bmod 2^{n}\right)+a 2^{n}+\left(\left(x 2^{l}+s\right) \bmod 2^{n}\right) \\
& \equiv b 2^{n}+\left(g_{s}(x) \bmod 2^{n}\right) \quad\left(\bmod 2^{n+N}\right)
\end{aligned}
$$

Поэтому условие (4) выполняется для всех допустимых значений $a, b$ и $\alpha_{2}\left(g_{s}\right)=1$.

Определение 7. Под $n$-граммой будем понимать $\alpha \in\left\{0,1, \ldots, p^{n}-1\right\}, n \in \mathbf{N}$.

Определение 8. Будем говорить что $c \in \mathbf{Z}_{p}$ реализует $n$-грамму $\alpha$, если существует такое $l \in \mathbf{N}$, что $H_{p}(c, l, n)=\alpha$.

Теорема 11. Пусть $\mathbf{C} \in \mathbf{Z}_{2}$ таково, что $\mathbf{C}$ реализует нулевую п-грамму бесконечно много раз. Положим

$$
g_{s}(x)=\mathbf{F}_{\mathbf{C}}\left(x 2^{l}+s\right), \quad l \in \mathbf{N} \cup\{0\}, \quad s \in\left\{0,1, \ldots, 2^{l}-1\right\} .
$$

Тогда $\alpha_{2}\left(g_{s}\right)=1$ для всевозможных значений параметров l u s. 
Доказательство. По условию, для любого $N \in \mathbf{N}$ существует $n>3(3 N+l+2)+N$ такое, что

$$
H_{2}(\mathbf{C}, n-N, N) \equiv 0 \quad\left(\bmod 2^{N}\right) .
$$

По лемме 1 , для произвольных значений $a, b \in\left\{0,1, \ldots, 2^{N}-1\right\}$ существует $x_{0} \in \mathbf{Z} / 2^{n} \mathbf{Z}$ такой, что выполняется условие (4) для квадратичной функции $f_{s}(x)=\left(x 2^{l}+s\right)^{2}$. Рассмотрим при данном $x_{0}$ значение функции $g_{s}^{\prime}(x)=f_{s}(x) \vee \mathbf{C}$ :

$$
\begin{aligned}
g_{s}^{\prime}\left(x_{0}\right) & \equiv\left(b 2^{n-N+l}+\left(f_{s}\left(x_{0}\right) \bmod 2^{n-N+l}\right)\right) \vee\left(\mathbf{C} \bmod 2^{n+l}\right), \\
& \equiv b 2^{n-N+l}+\left(f_{s}\left(x_{0}\right) \bmod 2^{n-N+l}\right) \vee\left(\mathbf{C} \bmod 2^{n-N+l}\right), \\
& \equiv b 2^{n-N+l}+\left(g_{s}^{\prime}\left(x_{0}\right) \quad\left(\bmod 2^{n-N+l}\right)\right) \quad\left(\bmod 2^{n+l}\right)
\end{aligned}
$$

Поэтому $\alpha_{2}\left(g_{s}^{\prime}\right)=1$ и в соответствии с леммой 2 значение $\alpha_{2}\left(g_{s}\right)=1$.

Теорема 12. Пусть $\mathbf{C} \in \mathbf{Z}_{2}$ таково, что существует такое натуральное $N \in \mathbf{N}$, что $\mathbf{C}$ не реализует нулевую $N$-грамму, тогда $\alpha_{2}\left(\mathbf{F}_{\mathbf{C}}\right)=0$.

Доказательство. Пусть $b \equiv a\left(\bmod 2^{N+1}\right)$, тогда для того, чтобы выполнялось условие (4) для $\mathbf{F}_{\mathbf{C}}$, необходимо, чтобы оно выполнялось для функции $x^{2} \vee \mathbf{C}$ при

$$
a^{\prime} \equiv a \quad\left(\bmod 2^{N+1}\right), \quad b^{\prime} \equiv b-a \equiv 0 \quad\left(\bmod 2^{N+1}\right) .
$$

Но это невозможно, так как $\sum_{i=0}^{N} \delta_{k+i}(\mathbf{C}) \neq 0$ для всех $k \in \mathbf{N}$ и для любого $x$ выполняется неравенство $H_{2}\left(x^{2} \vee \mathbf{C}, k, N\right) \neq 0$. Следовательно, условие (4) не выполняется для всех пар точек вида $(a, 0)$. Из закона 0 и 1 (см. теорему 7) непосредственно следует утверждение теоремы.

Следствие 4. Пусть $\mathbf{C} \in \mathbf{Z}_{2} \cap\left(\mathbf{Q} \backslash \mathbf{N}_{0}\right)$, тогда $\alpha_{2}\left(\mathbf{F}_{\mathbf{C}}\right)=0$.

Следствие 5. Пусть $\mathbf{C} \in \mathbf{Z}_{2}$ является отрицательным цุельмм числом, тогда $\alpha_{2}\left(\mathbf{F}_{\mathbf{C}}\right)=0$.

\section{6. Свойства множества предельных точек $\mathscr{P}^{2}\left(\mathbf{F}_{\mathbf{C}}\right)$}

В случае, если $\alpha_{2}\left(\mathbf{F}_{\mathbf{C}}\right)=0$, особый интерес представляет множество предельных точек $\mathscr{P}^{2}\left(\mathbf{F}_{\mathbf{C}}\right)$, так как, зная это множество в любой момент времени, можно проверять в первую очередь именно их $\varepsilon$-окрестности при данном входе $x$.

Определение 9. Для $f: \mathbf{Z}_{p} \rightarrow \mathbf{Z}_{p}$ обозначим через $\mathscr{L}^{k}(f)$ множество предельных неизолированных точек $\mathscr{P}^{k}(f)$.

Далее будет рассмотрена структура множества $\mathscr{L}^{2}\left(\mathbf{F}_{\mathbf{C}}\right)$ и оценена размерность Хаусдорфа (также известная как размерность Хаусдорфа-Безиковича) этого множества, которая может быть использована для более точного описания структуры измеримых множеств с нулевой мерой Лебега.

Определение 10. Для всех $\alpha \in[0,1]$

$$
\begin{aligned}
l_{\alpha}(x) & = \begin{cases}x+\alpha, & \text { при } x+\alpha \leqslant 1, \\
x+\alpha-1 & \text { при } x+\alpha>1,\end{cases} \\
A_{\alpha} & =\left\{\left(x, l_{\alpha}(x)\right): x \in[0,1]\right\} \subset[0,1]^{2} .
\end{aligned}
$$


Определение 11. Пусть $\mathbf{C} \in \mathbf{Z}_{2} \cap \mathbf{Q}$ представимо в виде

$$
\mathbf{C}=c \sum_{i=0}^{\infty} 2^{l i}, \quad l \in \mathbf{N}, \quad c \in\left\{1,2, \ldots, 2^{l}-1\right\},
$$

тогда обозначим

$$
\operatorname{Coord}_{\mathbf{C}}=\left\{\alpha_{j}: \alpha_{j}=\sum_{i=0}^{\infty} \frac{\alpha_{i}^{j}}{2^{i+1}} \in[0,1], \alpha_{i}^{j} \in\{0,1\}, j \in\{0,1, \ldots, l-1\}\right\},
$$

для которого при всех $t, j \in\{0,1, \ldots, l-1\}$, если $\delta_{(l-1-j-t)(\bmod l)}(c)=1$, то для всех $s \in \mathbf{N} \cup\{0\}$ значение $\alpha_{l s+t}^{j}=0$, все остальные $\alpha_{i}^{j}$ принимают произвольные значения из $\{0,1\}$.

Теорема 13. Пусть $\mathbf{C} \in \mathbf{Z}_{2} \cap \mathbf{Q}$, тогда

$$
\mathscr{L P P}^{2}\left(\mathbf{F}_{\mathbf{C}}\right)=\bigcup_{\alpha \in \operatorname{Coord}_{\mathbf{C}}} A_{\alpha}
$$

Доказательство. Пусть $(u, v) \in \mathscr{L}^{2}\left(\mathbf{F}_{\mathbf{C}}\right)$ - произвольная неизолированная предельная точка множества $\mathscr{P}^{2}\left(\mathbf{F}_{\mathbf{C}}\right)$. Тогда существует последовательность $\left(x_{i}, \mathbf{F}_{\mathbf{C}}\left(x_{i}\right) \bmod 2^{n_{i}}\right)$, $x_{i} \in\left\{0,1, \ldots, 2^{n_{i}}-1\right\}, n_{i+1}>n_{i}$, такая, что

$$
\lim _{i \rightarrow \infty}\left(\frac{x_{i}}{2^{n_{i}}}, \frac{\mathbf{F}_{\mathbf{C}}\left(x_{i}\right) \bmod 2^{n_{i}}}{2^{n_{i}}}\right)=(u, v) .
$$

Так как

$$
\mathbf{C} \equiv\left(c \bmod 2^{n_{i} \bmod l}\right) 2^{n_{i}-\left(n_{i} \bmod l\right)}+c \sum_{j} 2^{l j} \quad\left(\bmod 2^{n_{i}}\right)
$$

для любого $n_{i}$, мы видим, что

$$
\left(x_{i}^{2} \vee \mathbf{C}\right) \bmod 2^{n_{i}}=\left\lfloor\alpha 2^{n_{i}}\right\rfloor
$$

для некоторого $\alpha \in$ Coord $_{\mathbf{C}}$. Следовательно, точка $(u, v)$ лежит на некоторой прямой $l_{\alpha}$. Из этого можно заключить, что

$$
\mathscr{L} \mathscr{P}^{2}\left(\mathbf{F}_{\mathbf{C}}\right) \subseteq \bigcup_{\alpha \in \operatorname{Coord}_{\mathbf{C}}} A_{\alpha}
$$

Обратно, для каждой точки произвольной прямой $l_{\alpha} \subset A_{\alpha}$ при $\alpha \in$ Coord $_{\mathbf{C}}$ можно сопоставить сходящуюся к ней последовательность $\left(x_{i} / 2^{n_{i}}, \mathbf{F}_{\mathbf{C}}\left(x_{i}\right) \bmod 2^{n_{i}} / 2^{n_{i}}\right)$.

Теорема 14. Пусть $\mathbf{C} \in \mathbf{Z}_{2} \cap \mathbf{Q}$ представимо в виде

$$
\mathbf{C}=c \sum_{i=0}^{\infty} 2^{l i}, \quad l \in \mathbf{N}, \quad c \in\left\{1,2, \ldots, 2^{l}-1\right\},
$$

тогда размерность Хаусдорфа множества $\mathscr{L P}^{2}\left(\mathbf{F}_{\mathbf{C}}\right)$ равна $\operatorname{wt}(c) / l$, где

$$
\operatorname{wt}(c)=\sum_{i=0}^{l-1} \delta_{i}(c)
$$

- вес Хэмминга. 
Доказательство. Пусть $\Theta_{j}-$ покрытие множества $\mathscr{L P}^{2}\left(\mathbf{F}_{\mathbf{C}}\right)$,

$$
\Theta_{j}=\bigcup_{\beta}\left\{A_{\beta+\xi}: \xi \in\left[0,1 / 2^{l(j+1)}\right]\right\}=\bigcup_{\beta} B_{\beta}
$$

для всех $\beta \in[0,1], \beta 2^{l(j+1)}=c \sum_{i=0}^{j-1} 2^{l i} \in \mathbf{N}$, для которого по построению

$$
\Theta_{0} \subset \Theta_{1} \subset \ldots \subset \Theta_{n} \subset \ldots \subset \mathscr{L P}^{2}\left(\mathbf{F}_{\mathbf{C}}\right) \text {. }
$$

С учетом того, что мера Лебега элементов $B_{\beta}$ равна $2^{-l(j+1)}$ и $\Theta_{j}$ является $\varepsilon$-покрытием $\mathscr{L}^{2}\left(\mathbf{F}_{\mathbf{C}}\right)$ при $\varepsilon=2^{-l(j+1)}$, значение $\alpha$-меры Хаусдорфа удовлетворяет неравенству

$$
\begin{aligned}
M_{\alpha}\left(\mathscr{L} \mathscr{P}^{2}\left(\mathbf{F}_{\mathbf{C}}\right)\right) & \leqslant \lim _{j \rightarrow \infty} \sum_{\beta}\left(2^{-l(j+1)}\right)^{\alpha}=\lim _{j \rightarrow \infty} 2^{w t(c)(j+1)}\left(2^{-l(j+1)}\right)^{\alpha} \\
& =\lim _{j \rightarrow \infty} 2^{(j+1)(w t(c)-\alpha l)} .
\end{aligned}
$$

Для любого минимального $\varepsilon$-покрытия $\Theta_{\varepsilon}^{\prime}$ множества $\mathscr{L} \mathscr{P}^{2}\left(\mathbf{F}_{\mathbf{C}}\right)$ можно найти натуральное $j^{\prime}$ такое, что $\Theta_{j^{\prime}} \subset \Theta_{\varepsilon}^{\prime}$ и

$$
M_{\alpha}\left(\mathscr{L} \mathscr{P}^{2}\left(\mathbf{F}_{\mathbf{C}}\right)\right)=\lim _{\varepsilon \rightarrow 0+} \Theta_{\varepsilon}^{\prime} \geqslant \lim _{j^{\prime} \rightarrow \infty} \sum_{\beta}\left(2^{-l\left(j^{\prime}+1\right)}\right)^{\alpha} .
$$

Отсюда следует, что

$$
M_{\alpha}\left(\mathscr{L} \mathscr{P}^{2}\left(\mathbf{F}_{\mathbf{C}}\right)\right)=\lim _{j \rightarrow \infty} 2^{(j+1)(\mathrm{wt}(c)-\alpha l)} .
$$

При $\alpha>\alpha_{0}=\operatorname{wt}(c) / l \alpha$-мера равна нулю. При $\alpha<\alpha_{0}=\operatorname{wt}(c) / l \alpha$-мера равна $\infty$. Таким образом, $\alpha_{0}$ является размерностью Хаусдорфа.

\section{7. Заключение}

В работе были детально рассмотрены свойства гаммы, вырабатываемой ГПСЧ КлимоваШамира, и показаны их неудовлетворительные статистические свойства. Некоторые исследователи, отмечая слабые криптографические свойства генератора при $c<2^{n / 3}$, предлагают выбирать значения $c \geqslant 2^{n / 3}$ (см. раздел 2). В данном случае, в соответствии с теоремой 2 , множество пар точек $\left(x, F_{c}(x)\right)$ распадается на полосы. Техника, предложенная в теореме, наглядно продемонстрирована в примере 2.

Показано (см. следствие 2), что в случае, если $n=32$, требуется не более 9 различных пар $\left(x_{i}, F_{c}\left(x_{i}\right)\right)$ для определения значения $c$ с вероятностью $P \geqslant 0,999$. Для изучения свойств распределения пар $(x, f(x))$ Т-функции $f: \mathbf{Z} / p^{n} \mathbf{Z} \rightarrow \mathbf{Z} / p^{n} \mathbf{Z}$ продемонстрирован подход, впервые описанный в 2009 г. в [6], включающий исследование этого распределения в более общем случае, с использованием продолжения Т-функции $f$ на множество целых $p$-адических чисел $F: \mathbf{Z}_{p} \rightarrow \mathbf{Z}_{p}$. В рамках этого подхода исследуется мощность множества состояний $\mathcal{N}$ автомата Мили $U=\left\langle\mathcal{K}, \mathcal{N}, \mathcal{M}, f, F, u_{0}\right\rangle$, для автоматной функции $\Psi_{\iota}$ которого существует взаимно однозначное соответствие с $F$.

Если существует такой автомат с конечным числом состояний, то в общем случае ГПСЧ или поточный шифратор не может считаться хорошим.

Для ГПСЧ Климова-Шамира $\mathbf{F}_{\mathbf{C}}$, в зависимости от значения константы $\mathbf{C} \in \mathbf{Z}_{2}$, полностью описаны условия, при которых существует и не существует соответствующий конечный автомат $и$. 
Определены условия, при которых $\mathbf{F}_{\mathbf{C}}$ позволяет приблизить произвольную точку $(a, b) \in[0,1]^{2}$ точкой $\left(x / p^{n},\left(\mathbf{F}_{\mathbf{C}}(x) \bmod p^{n} / p^{n}\right)\right.$, вырабатываемой $\mathbf{F}_{\mathbf{C}}$ на входе длины $n$, что является одной из характеристик качества гаммы при использовании $\mathbf{F}_{\mathbf{C}}$ как генератора псевдослучайных чисел. В терминах монографии [6] проведен анализ функции $\alpha_{2}\left(\mathbf{F}_{\mathbf{C}}\right)$ - меры Лебега замыкания проекции на $[0,1]^{2}$ множества всех достижимых точек.

Отметим, что в поточных шифраторах в целях увеличения быстродействия используются преобразования $F: \mathbf{Z}_{p} \rightarrow \mathbf{Z}_{p}$, удовлетворяющие условию Липшица с константой 1 , соответствующие конечным автоматам. Например, к их числу относятся поточный шифратор АВС и семейство TSC-і. В этом случае, согласно теореме 11.10 из [6], всегда $\alpha_{2}(F)=0$.

Для оценки структуры множества $\mathscr{P}^{2}(F)$ предложен подход на примере $\mathbf{F}_{\mathbf{C}}$, связанный с оценкой множества всех предельных неизолированных точек $\mathscr{L P}^{2}(F)$ с использованием размерности Хаусдорфа.

Отметим, что использование операции возведения в квадрат сравнивает по скорости работы $F_{c}$ и квадратичные генераторы. Теоретические исследования распределения пар $\left(x_{i}, x_{i+1}\right)$, троек $\left(x_{i}, x_{i+1}, x_{i+2}\right)$, разреженных пар $\left(x_{i}, x_{i+2}\right)$ проводились в классе полиномов степени 2 (см. [10,8,9] соответственно) в середине 90-х и позволяют строить квадратичные конгруэнтные генераторы с равномерным распределением.

Автор выражает признательность В. С. Анашину за ценные советы и поддержку при написании статьи.

\section{Список литературы}

1. Анашин В. С., Равномерно распределенные последовательности целых $p$-адических чисел. Математические заметки (1994) 55, №2, 3-46.

2. Anashin V. S., Uniformly distributed sequences over p-adic integers. In: Proc. Intern. Conf. "Number theoretic and algebraic methods in computer science" (van der Poorten A. J., Shparlinsky I., Zimmer H. G., eds.). World Scientific, Singapore, 1995, pp. 1-18.

3. Anashin V. S., Uniformly distributed sequences in computer algebra, or how to construct program generators of random numbers. J. Math. Sci. (1998) 89, №4, 1355-1390.

4. Анашин В. С., Равномерно распределенные последовательности целых $p$-адических чисел. Дискретная математика (2002) 14, №4, 3-64.

5. Anashin V. S., Ergodic transformations in the space of $p$-adic integers. AIP Conf. Proc. (2006) 826, $3-24$.

6. Anashin V. S., Khrennikov A. Yu., Applied algebraic dynamics. de Gruyter, Berlin, 2009.

7. Bénony V., Recher F., Wegrzynowski E., Fontaine C., Cryptanalysis of a particular case of KlimovShamir Pseudo-Random Generator. Lect. Notes Computer Sci. (2004) 3486, 313-322.

8. Eichenauer-Herrmann J., Quadratic congruential pseudorandom numbers: Distribution of triples. $J$. Comput. Appl. Math. (1995) 62, 239-253.

9. Eichenauer-Herrmann J., Quadratic congruential pseudorandom numbers: Distribution of lagged pairs. J. Comput. Appl. Math. (1997) 79, 75-85.

10. Emmerich F., Equidistribution properties of quadratic congruential pseudorandom numbers. J. Comput. Appl. Math. (1997) 79, 207-214.

11. Klimov A., Applications of T-functions in cryptography, PhD Thesis, Dept. Appl. Math. Computer Sci. Weizmann Institute of Science, Rehovot, 2005.

12. Klimov A., Shamir A., A new class of invertible mappings. Lect. Notes Comput. Sci. (2002) 2523, 470-483. 
13. Klimov A., Shamir A., Cryptographic applications of T-functions. Lect. Notes Comput. Sci. (2003) 3006, 248-261.

14. Klimov A., Shamir A., New cryptographic primitives based on multiword T-functions. Lect. Notes Comput. Sci. (2004) 3017, 1-15.

15. Klimov A., Shamir A., New applications of T-functions in block ciphers and hash functions. Lect. Notes Comput. Sci. (2005) 3557, 18-31.

16. Molland H., Helleseth T., A linear weakness in the Klimov-Shamir T-function. In: Proc. ISIT'2005. IEEE, 2005, pp. 1106-1110.

17. Molland H., Helleseth T., Linear properties in T-functions. IEEE Trans. Inform. Theory (2006) 52, №11, 5151-5157.

18. Wang J.-S., Qi W.-F., Linear equation on polynomial single cycle T-functions. Lect. Notes Comput. Sci. (2008) 4990, 256-270.

Статья поступила 16.04.2010. 